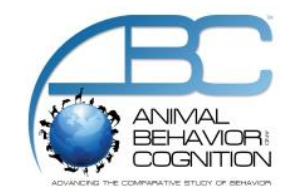

\title{
Perception of Angle in Visual Categorization by Pigeons (Columba livia)
}

\author{
Walter T. Herbranson*, Elizabeth Karas, and Glendon Hardin
}

Whitman College, Walla Walla, Washington, USA

*Corresponding author (Email: herbrawt@whitman.edu)

Citation - Herbranson, W. T., Karas, E., \& Hardin, G. (2017). Perception of angle in visual categorization by pigeons (Columba livia). Animal Behavior and Cognition, 4(3), 286-300. https://doi.org/10.26451/abc.04.03.07.2017

\begin{abstract}
Pigeons are capable of learning to categorize stimuli based on visual features, and often reach levels of accuracy comparable with humans. Nevertheless, recent research has suggested that the cognitive processes behind categorization in pigeons and humans may not always be the same. Pigeons learned a categorization task in which they categorized either Shepard circles varying in size and orientation of a radial line (stimuli that are frequently used in research on human categorization), or moving dots varying in speed and direction of travel (stimuli that have been successfully used to study pigeon categorization). Even though categories were balanced so that the angles of orientation of Shepard circles matched the directions of travel for moving dots, birds failed to learn categories based on the former but not the latter. Results suggest that information about angle as a direction of travel may be more important for pigeons than information about angle of orientation.
\end{abstract}

Keywords - Categorization, Perception, Pigeon, Motion, Orientation

Categorization seems to be a fundamental cognitive process, essential to survival for a wide variety of animals (Jitsumori \& Delius, 2001). It allows an animal to recognize the functional equivalence of an entire class of objects, despite tremendous variation in the sensory impressions they make (based on natural variability between exemplars within a category, as well as variables like viewing angle, lighting conditions, distance, and background). Despite the fact that many animals learn and use categories effectively, they may not always do so in the same way. For example, Smith et al. (2012) found that pigeons differed from primate species (including humans) when categorizing by not analyzing multidimensional stimuli into their component elements. It is important to note though, that whereas their pigeons did not learn categories in the same way as primates, they did successfully learn to categorize stimuli accurately. These kinds of inter-species differences illustrate the value of multiple research approaches and methods to studying categorization; one method may highlight an important difference that another fails to reveal.

There are numerous ways to study visual categorization in a laboratory setting. Perhaps the most direct involves presentation of realistic, detailed stimuli such as photographs of exemplars from realworld categories like "tree" or "human" (e.g., Herrnstein \& Loveland, 1964). This approach is appealing in the sense that such realistic stimuli capture the complex category structures that animals encounter in the wild. At the same time, it is not always clear what a successful animal has learned. Specifically, one cannot be certain which feature or features of a stimulus caused an animal to make a particular categorization response. Other investigations may use categories created by the investigators so that all 
exemplars possess some unambiguous feature or collection of features (e.g., Lea et al., 2009; Wills et al., 2009). The advantages of this approach are complementary. Although it is easier to pinpoint the relevant features, it may be the case that the stimuli do not fully represent the complexity of the natural world.

Intermediate methods (e.g., Chase \& Heinemann, 1972; Herbranson, Fremouw, \& Shimp, 1999, 2002) may capture some of the desirable characteristics (and perhaps some of the disadvantages) of both approaches. Categories can be created using continuous, multivariate distributions, meaning that a virtually unlimited number of unique exemplars can be generated. Stimuli toward the center of a distribution have a higher probability of occurrence, corresponding to the more typical members of a category. Stimuli further away from the center are less likely to occur given the category, and correspond to more rare or anomalous exemplars that are nonetheless category members. Figure 1 shows a schematic of two category distributions, each defined along the same two continuous stimulus dimensions. The primary benefit of this approach (sometimes called the randomization procedure) is that it captures the variability and overlap of features seen in natural categories in a way that is still mathematically precise. Furthermore, there are no necessary and sufficient features required for category membership that might lead to a simple memorization strategy. In fact, any particular value along either dimension could occur in members of both categories.
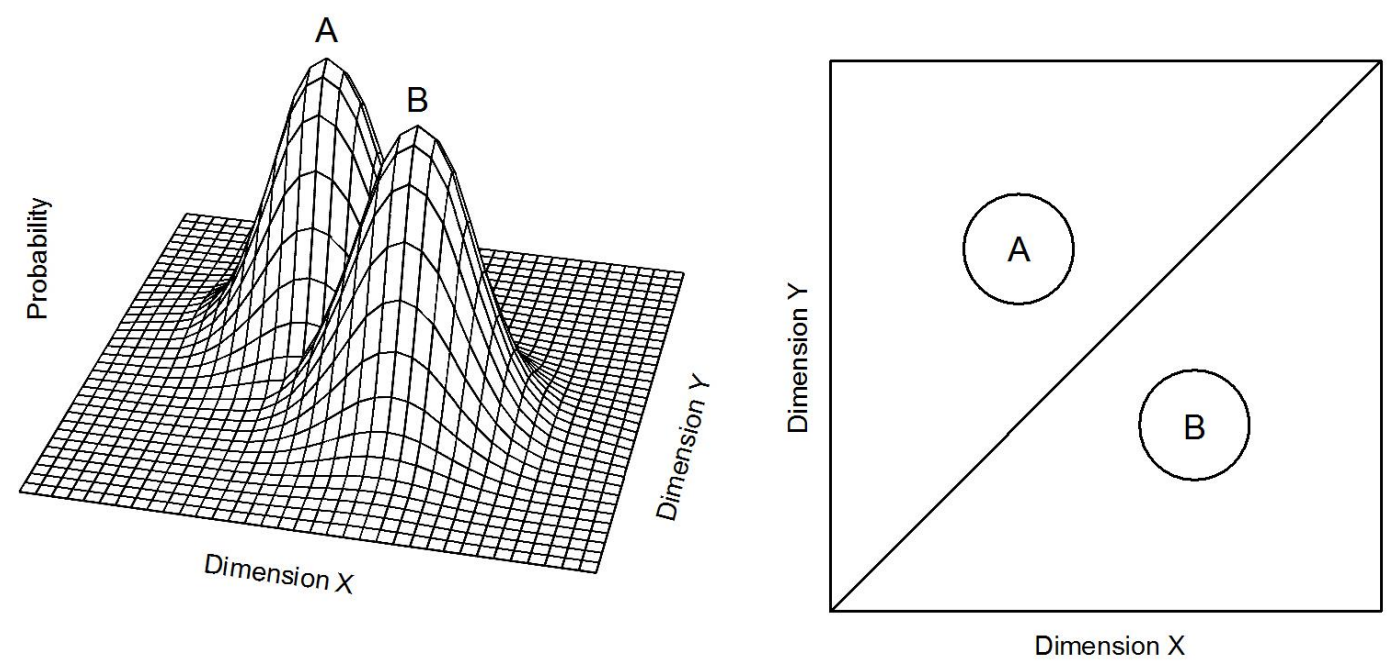

Figure 1. Left Panel: Bivariate normal distributions like those used to generate stimuli in experiments using the randomization procedure. Right Panel: 2-dimensional stimulus space representing categories depicted in the left panel.

Similar methods have been extensively used in the study of human categorization (e.g., Ashby \& Gott, 1988), backed up by impressively sophisticated methods of data analysis. Responses from each participant are analyzed to yield a decision bound that efficiently describes their response strategy. A decision bound is a line that passes through the imaginary two-dimensional stimulus space in which the presented stimuli are defined. Each individual participant will have a unique decision bound that best describes their performance, in that it separates stimuli that the participant categorized differently. A participant's decision bound may be optimal, matching the decision bound of a hypothetical optimal classifier that always maximizes correct categorizations. Alternatively, it may be suboptimal, yielding a consistent and predictable pattern of errors on specific kinds of stimuli located in specific regions of the stimulus space.

The use of decision bounds is further appealing because it can give insight into other cognitive processes. For example, some pairs of stimulus dimensions are said to be separable, meaning that a response can be made based on one stimulus dimension by itself, without interference from a second dimension (Shepard, 1964). Others may be integral, meaning that one dimension influences how a second is perceived (Lockhead, 1966). In the former case, selective attention to a single stimulus dimension is relatively easy, and would be indicated by a decision bound that lies parallel to one axis of 
the stimulus space. Integral dimensions on the other hand, make selective attention difficult, and yield decision bounds that are not parallel to either axis.

This method for studying categorization is quite flexible, and can be implemented using a tremendous variety of stimulus types. Human research has used numerous kinds of stimuli (each varying on two continuous dimensions), including rectangles (height and width; Ashby \& Maddox 1992), Shepard circles (size and orientation; Ashby \& Maddox, 1990), color patches (brightness and saturation; Maddox \& Dodd, 2003), and sine-wave gratings (orientation and spatial frequency; Maddox, Ashby, \& Bohil, 2003). Similarly, pigeon research has used a variety of stimulus types, including rectangles (height and width; Herbranson et al., 1999), movement vectors (speed and direction; Herbranson, et al., 2002), and sine-wave gratings (orientation and spatial frequency; Smith et al., 2011). In each of these cases, pigeons have been able to learn selective attention categories (those based on a single stimulus dimension), as well as information integration categories (those based on both stimulus dimensions).

Whereas the method has been quite useful for studying categorization in both humans and pigeons, most individual investigations have focused on a single species. For example, although categorization tasks using Shepard circles have contributed greatly to an understanding of dimensional separability in human categorization, they have not been used in the same way to investigate pigeon categorization. There is reason to suspect that pigeons could learn to categorize Shepard circles, and contribute to a similar understanding of dimensional separability in pigeons. Herbranson et al. (1999) had pigeons categorize rectangles varying in height and width, and in one condition the two categories corresponded to rectangles with larger or smaller areas (similar to the size dimension in Shepard circles). Later, the same team (Herbranson et al., 2002) investigated pigeons' categorization of moving targets in terms of speed and angle of movement, and demonstrated that pigeons could acquire categories based on angle, the second dimension by which Shepard circles vary. These prior investigations also provide some information about how best to scale each dimension in order to ensure that pigeons' visual acuity is adequate to make the necessary between-category discriminations.

Here we investigated pigeons' categorization of Shepard circles, based on size, orientation, or a combination of the two. If pigeons can selectively attend to a single relevant dimension, the two singledimension conditions should yield high categorization accuracy and decision bounds perpendicular to the relevant axis. Similarly, if pigeons can integrate information from both dimensions, integration conditions should yield high categorization accuracy and decision bounds that are not perpendicular to either axis. For comparison, we trained additional pigeons on categories of moving dots, based on speed, direction of movement, or both. Pigeons have successfully learned to categorize these kinds of stimuli, and categories were constructed so that there was a close match between stimuli in parallel Shepard circle and movement conditions. Inclusion of these comparison conditions involving stimuli that pigeons have successfully learned to categorize in previous research should provide some valuable context regarding the kinds of features pigeons can effectively use in categorization, as well as the relative difficulty of learning different kinds of categories.

\section{Animals}

\section{Method}

Twenty-four White Carneau pigeons (Columba livia) were obtained from Double-T Farm (Glenwood, IA). Each was maintained at approximately $80-85 \%$ of its free-feeding weight (Poling, Nickel \& Alling, 1990), with supplemental grain provided as needed in home cages after daily experimental sessions. Each pigeon was housed individually in a metal pigeon cage with free access to water and grit, in a colony room with a $14: 10 \mathrm{hr}$ light/dark cycle. All experimental sessions took place during the light cycle at approximately the same time 5 or 6 days per week. 


\section{Apparatus}

Two custom operant chambers were used (internal dimensions $30 \times 43 \times 43 \mathrm{~cm}$ ). In the front wall of each chamber was a 15" flat panel computer monitor (Dell Inc., Round Rock, TX) with an infrared touch frame (Carroll Touch, Round Rock, TX). Ten $\mathrm{cm}$ below the monitor was a feeder (BRS/LVE, Laurel, MD) through which birds could gain access to mixed grain via a $6 \times 7 \mathrm{~cm}$ opening. Each chamber was interfaced to a computer that controlled all experimental events, presented stimuli, recorded data, and computed daily statistics. Each chamber was equipped with a ventilation fan and a speaker that produced white noise throughout each experimental session.

\section{Stimuli}

Stimuli were either Shepard circles, or dots traveling along a movement vector, depending on the experimental condition.

Shepard Circles. Shepard circle stimuli consisted of a closed white semicircle plus a green radial line connecting two points on the semicircle. The white (Hexadecimal color code \#FFFFFF) and green (hexadecimal color code \#00FF00) elements were the same brightness on the screen. The flat diameter of the semicircle was always oriented so that it was parallel with the bottom edge of the screen. The lines defining the semicircle were white, and approximately $2 \mathrm{~mm}$ thick. Within the semicircle was a green radius, approximately $2 \mathrm{~mm}$ thick extending from the center of the circle's flat base to a point on its curved edge. The size of the semicircle and the angle of the radial line varied from trial to trial, and stimuli were drawn from one of the two bivariate normal distributions that defined categories for each condition. Angle and size were both measured in units that were convenient to the program structure of the control software. One unit of angle was equal to approximately 1 degree, with 90 being vertical on the screen. Higher numbers corresponded to lines that tilted toward the right; lower numbers to the left. One unit of size was equal to approximately $1 \mathrm{~mm}$ of a circle's radius as displayed on the screen.

Vectors. Vector stimuli consisted of solid white dots approximately $1 \mathrm{~cm}$ in diameter, moving across the screen at a constant speed and direction. A white dot initially appeared stationary against the background of an otherwise blank screen. A single peck to the dot initiated movement in a straight line and at a constant speed. The angle and speed of movement varied across trials in the same way that angle and size of Shepard circles varied from trial to trial, and were drawn from one of the two bivariate normal distributions that defined categories for each condition. Angle and speed of movement were both measured in units that were convenient to the program structure of the control software and corresponded closely to the units that defined Shepard circles. One unit of angle was equal to approximately 1 degree, with 90 being straight toward the top of the screen. Higher numbers produced movement at proportionally increasing angles toward the right side of the screen; lower numbers toward the left. A moving stimulus travelled at a constant rate, covering the specified distance (in $\mathrm{mm}$ ) in $2.0 \mathrm{~s}$. Thus, one unit of speed was equal to $0.5 \mathrm{~mm} / \mathrm{sec}$.

\section{Conditions}

Birds were arbitrarily assigned to one of six conditions, differing based on stimulus type (Shepard circles or vectors) and the stimulus component or components diagnostic of category membership (angle only, size/speed only, or integration). The combination of these two factors yielded six conditions: Shepard circles angle-only (SA), Shepard circles size-only (SS), Shepard circles integration (SI), vectors angle-only (VA), vectors speed-only (VS), and vectors integration (VI). Figure 2 shows the prototypical stimuli (those at the center of each category distribution, and thus, most likely to occur) for the two categories in each condition.

Angle only. In angle-only conditions the distribution that defined category A had a mean size (Shepard circles) or speed (vectors) of $80(S D=15)$ and a mean angle of $100(S D=1)$. Category B had a 
mean size or speed of $80(S D=15)$ and a mean angle of $80(S D=1)$. Covariance between the two dimensions was 0 .

Size/speed-only. In size/speed-only conditions, the distribution that defined category A had a mean size or speed of $90(S D=1)$ and a mean angle of $90(S D=15)$. Category B had a mean size or speed of $70(S D=1)$ and a mean angle of $90(S D=15)$. Covariance between the two dimensions was 0 .

Integration. In integration conditions, the distribution that defined category A had a mean size or speed of $73(S D=10)$ and a mean angle of $97(S D=10)$. Category B had a mean size or speed of 87 $(S D=10)$ and a mean angle of $83(S D=10)$. Covariance between the two dimensions was 110 .

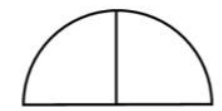

Category A Size $M=90, S D=1$ Angle $M=90, S D=15$

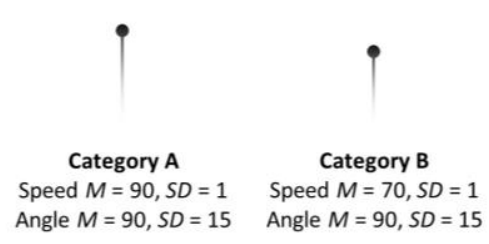

Size / Speed

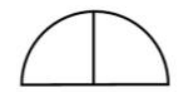

Category B Size $M=70, S D=1$ Angle $M=90, S D=15$

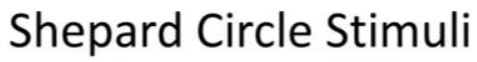

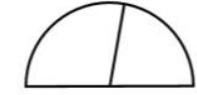

Category A Size $M=80, S D=15$ Angle $M=100, S D=1$

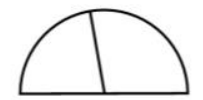

Category B Size $M=80, S D=15$ Angle $M=80, S D=1$

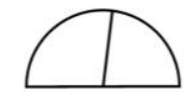

Category A Size $M=73, S D=10$ Angle $M=97, S D=10$

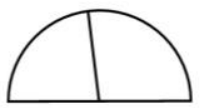

Category B Size $M=87, S D=10$ Angle $M=83, S D=10$

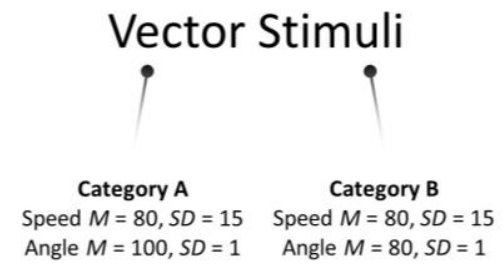

Angle

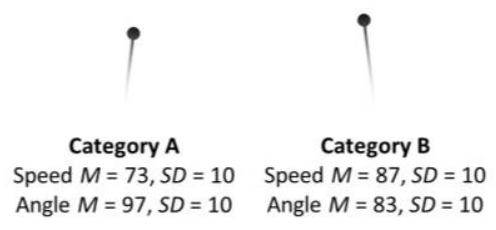

Integration

Figure 2. Prototypical stimuli from each of the categories used in six conditions. Each pair of stimuli depicts the most probable stimulus for each of the two categories in a given condition. Top panels: Shepard circle stimuli. Values correspond to approximately $1 \mathrm{~mm}$ (size) and 1 degree (angle). Bottom panels: vector stimuli. Values correspond to approximately $1 \mathrm{~mm}$ (size) and 1 degree (angle). The gradient line is to indicate direction of motion, and was not visible during a trial. A stimulus traversed the specified distance in 2.0 seconds.

\section{Procedure}

Birds completed 60 days of training with 80 trials per day, except for the first five days, during which the number of trials was gradually increased from an initial value of 10 .

Pretraining. All birds were pretrained in sessions consisting successively of habituation, magazine training, and autoshaping (Brown \& Jenkins, 1968) until consistent responding was achieved. Stimuli during autoshaping were $3 \times 3 \mathrm{~cm}$ colored squares (blue, green, red, or white) presented in three possible screen locations (randomly determined on each trial), evenly spaced in a horizontal row and centered vertically on the monitor.

Trial Organization. Each trial consisted of an inter-trial interval (ITI), presentation of an orienting cue, a required peck to the orienting cue, presentation of a stimulus, a categorization response, and either reinforcement or a correction procedure, depending on the accuracy of the categorization response.

The ITI was $5 \mathrm{~s}$, during which the houselight remained on and the monitor was blank. Following the ITI, an orienting cue was presented. The cue was a $3 \times 3 \mathrm{~cm}$ green square, presented at the center of the monitor. Any peck to the orienting cue initiated presentation of a stimulus generated from the relevant distribution. The stimulus was centered at a randomly determined location within a $5 \mathrm{x} 5 \mathrm{~cm}$ region at the center of the screen (in order to undermine the effectiveness of response strategies based on stimulus features appearing in fixed screen locations). Following a 5-s observation period (Shepard circle 
conditions) or $2 \mathrm{~s}$ of movement (vector conditions), two $3 \times 3 \mathrm{~cm}$ square response cues appeared: a red square located $4 \mathrm{~cm}$ from the right edge of the screen, and a blue square located $4 \mathrm{~cm}$ from the left edge of the screen. The first peck to either of these cues resulted in either reinforcement or a correction procedure. Pecks to the red square were reinforced if the preceding stimulus was drawn from Category A, and pecks to the blue square were reinforced if the preceding stimulus was drawn from Category B. Reinforcement consisted of approximately $3 \mathrm{~s}$ access to mixed grain (times varied from bird to bird, in order to maintain individual running weights).

If a response was incorrect, a correction procedure began, during which the houselight switched between on and off every $0.5 \mathrm{~s}$ for $10 \mathrm{~s}$. Immediately after, the trial was repeated, beginning with the presentation of the same stimulus (with no ITI or orienting cue). A correct categorization response during the correction procedure resulted in reinforcement and advancement to the next trial. An incorrect response resulted in repetition of the correction procedure, and repetition continued until a correct response was produced, with no limit to the number of iterations. Only the first response on each trial was included in data analysis (no responses to correction trials were included, whether correct or incorrect).

\section{Results}

\section{Accuracy}

The percent of correct categorizations for each bird over the final 10 days of the experiment is shown in Tables 1 (Shepard circle conditions) and 2 (vector conditions), along with group means for each condition. Group means also appear in Figure 3. A 2 (stimulus type: Shepard circles, vectors) x 3 (category: angle-only, size/speed-only, integration) between-groups ANOVA revealed a significant main effect of stimulus type, $F(1,18)=41.186, p<0.001$, partial $\eta 2=0.696$; a significant main effect of category, $F(2,18)=25.481, p<0.001$, partial $\eta 2=0.739$; and a significant interaction between stimulus type and category, $F(2,18)=20.695, p<0.001$, partial $\eta 2=0.697$.

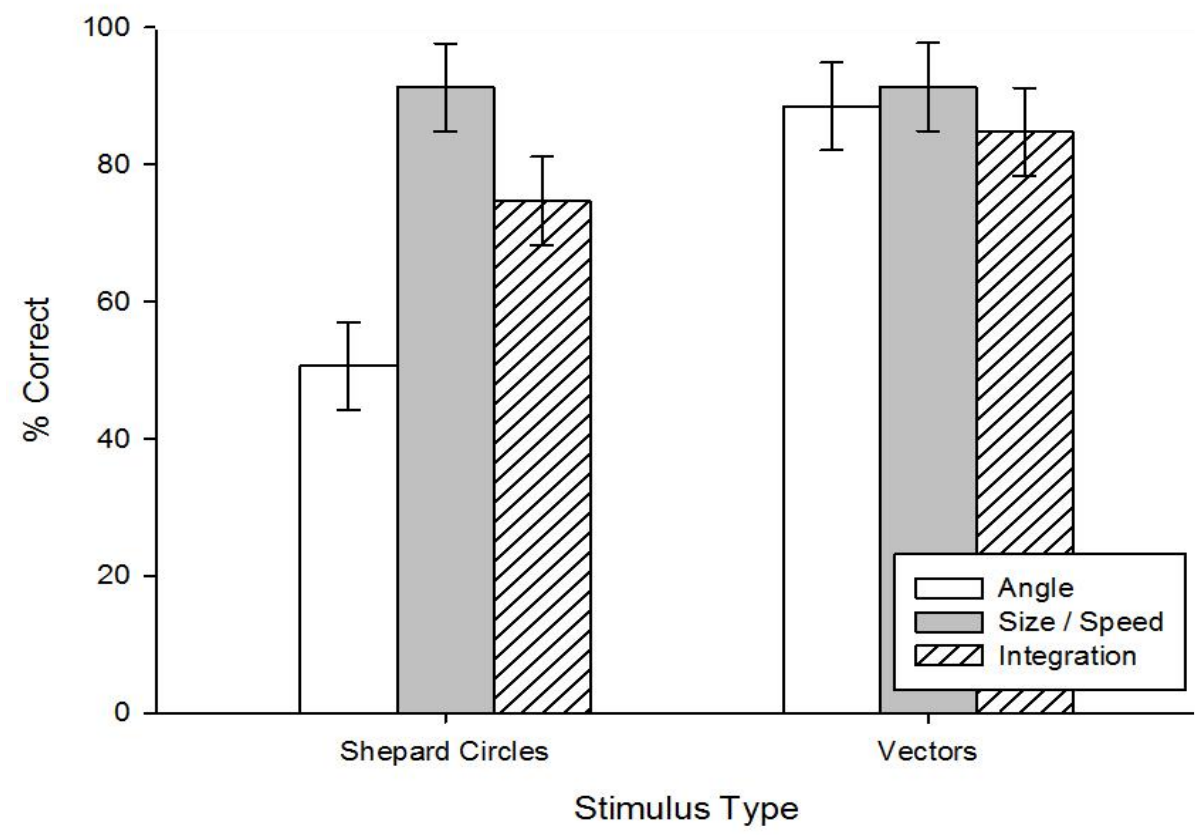

Figure 3. Average percent of correct categorization responses in each of six conditions. Error bars depict $95 \%$ confidence intervals. 
Table 1

Decision Bound Analyses for Pigeons in Conditions Using Shepard Circle Stimuli

\begin{tabular}{|c|c|c|c|c|c|}
\hline Condition SA (Angle Only) & Bird SA1 & Bird SA2 & Bird SA3 & Bird SA4 & Average \\
\hline$\%$ of correct responses & 51.3 & 51.9 & 49.4 & 49.7 & 50.6 \\
\hline \multicolumn{6}{|l|}{ Best-fitting bound } \\
\hline Slope & 0.40 & 1.00 & 5.00 & 0.80 & 1.71 \\
\hline Intercept & 86.25 & -59.25 & -435.00 & 49.50 & -89.63 \\
\hline \multicolumn{6}{|l|}{$\%$ of responses accounted for by } \\
\hline Best-fitting bound & 70.1 & 66.5 & 58.3 & 60.0 & 63.7 \\
\hline Best-fitting angle-only bound & 70.0 & 66.1 & 57.4 & 59.4 & 63.2 \\
\hline Angle $=90$ & 51.4 & 51.6 & 50.6 & 50.5 & $51.0 *$ \\
\hline Best-fitting size-only bound & 70.0 & 66.5 & 57.3 & 59.5 & 63.3 \\
\hline Size $=80$ & 50.1 & 54.3 & 50.2 & 50.5 & $51.3 *$ \\
\hline Condition SS (Size Only) & Bird SS1 & Bird SS2 & Bird SS3 & Bird SS4 & Average \\
\hline$\%$ of correct responses & 94.8 & 94.6 & 88.3 & 87.3 & 91.3 \\
\hline \multicolumn{6}{|l|}{ Best-fitting bound } \\
\hline Slope & -0.20 & 0.00 & 0.00 & -0.20 & -0.10 \\
\hline Intercept & 97.50 & 78.00 & 78.75 & 98.25 & 88.13 \\
\hline \multicolumn{6}{|l|}{$\%$ of responses accounted for by } \\
\hline Best-fitting bound & 94.9 & 94.6 & 88.3 & 87.4 & 91.3 \\
\hline Best-fitting angle-only bound & 53.0 & 52.6 & 55.3 & 59.5 & $55.1 *$ \\
\hline Angle $=90$ & 50.7 & 51.9 & 52.9 & 55.1 & $52.7 *$ \\
\hline Best-fitting size-only bound & 94.8 & 94.6 & 88.3 & 87.3 & 91.3 \\
\hline Size $=80$ & 94.8 & 94.6 & 88.3 & 87.3 & 91.3 \\
\hline Condition SI (Integration) & Bird SI1 & Bird SI2 & Bird SI3 & Bird SI4 & Average \\
\hline$\%$ of correct responses & 80.3 & 75.8 & 70.9 & 71.8 & 74.7 \\
\hline \multicolumn{6}{|l|}{ Best-fitting bound } \\
\hline Slope & -0.20 & 0.00 & 0.20 & 0.00 & 0.00 \\
\hline Intercept & 103.25 & 84.00 & 56.50 & 82.50 & 81.56 \\
\hline \multicolumn{6}{|l|}{$\%$ of responses accounted for by } \\
\hline Best-fitting bound & 83.9 & 79.6 & 76.9 & 79.5 & 80.0 \\
\hline Best-fitting angle-only bound & 70.6 & 55.6 & 56.8 & 58.4 & $60.4^{*}$ \\
\hline Angle $=90$ & 70.6 & 55.3 & 51.6 & 50.6 & $57.0 *$ \\
\hline Best-fitting size-only bound & 83.9 & 79.6 & 76.9 & 79.5 & 80.0 \\
\hline Size $=80$ & 82.6 & 78.8 & 75.0 & 77.4 & $78.5^{*}$ \\
\hline
\end{tabular}

Note. Bold headers indicate birds represented in Figure 4 for each condition. For ease of comparison, slope and intercept values for single-dimension categories (SA and SS) place the diagnostic dimension (angle and size, respectively) on the Y axis. Values for the integration condition place angle on the $\mathrm{Y}$ axis. * indicates an average significantly lower than that of the average bestfitting bound for the same condition $(p<0.05)$.

The main effect of stimulus type reflected more accurate categorization of vector stimuli $(M=$ 88.2, $S D=7.7)$ than Shepard circle stimuli $(M=72.2, S D=17.7)$. A post-hoc Tukey test compared the three category types, revealing significant differences between each pairing of angle-only $(M=69.5, S D$ $=20.5)$, size/speed-only $(M=91.3, S D=5.9$, and integration $(M=79.7, S D=8.9)$, all $p$ s $\leq 0.010$.

Figure 4 shows how one representative bird from each condition responded to each trial on the final day of the experiment. Birds represented are those that were closest to the overall mean percent correct for each condition. 
Table 2

Decision Bound Analyses for Pigeons in Conditions Using Vector Stimuli

\begin{tabular}{|c|c|c|c|c|c|}
\hline Condition VA (Angle Only) & Bird VA1 & Bird VA2 & Bird VA3 & Bird VA4 & Average \\
\hline$\%$ of correct responses & 92.5 & 81.3 & 90.0 & 90.0 & 88.5 \\
\hline \multicolumn{6}{|l|}{ Best-fitting bound } \\
\hline Slope & -0.20 & 0.20 & 0.00 & -0.20 & -0.05 \\
\hline Intercept & 100.50 & 58.50 & 80.00 & 102.00 & 85.25 \\
\hline \multicolumn{6}{|l|}{$\%$ of responses accounted for by } \\
\hline Best-fitting bound & 93.8 & 82.5 & 90.0 & 91.3 & 89.4 \\
\hline Best-fitting angle-only bound & 92.5 & 82.5 & 90.0 & 90.0 & 88.8 \\
\hline Angle $=90$ & 92.5 & 81.3 & 90.0 & 90.0 & 88.5 \\
\hline Best-fitting size-only bound & 56.3 & 60.0 & 57.5 & 58.8 & $58.2 *$ \\
\hline Size $=80$ & 50.0 & 51.9 & 56.9 & 58.1 & $54.2 *$ \\
\hline Condition VS (Speed Only) & Bird VS1 & Bird VS2 & Bird VS3 & Bird VS4 & Average \\
\hline$\%$ of correct responses & 80.0 & 93.8 & 92.5 & 98.8 & 91.3 \\
\hline \multicolumn{6}{|l|}{ Best-fitting bound } \\
\hline Slope & -0.20 & 0.20 & 0.20 & 0.00 & 0.05 \\
\hline Intercept & 100.25 & 75.50 & 73.00 & 80.00 & 82.19 \\
\hline \multicolumn{6}{|l|}{$\%$ of responses accounted for by } \\
\hline Best-fitting bound & 93.8 & 95.0 & 92.5 & 98.8 & 95.0 \\
\hline Best-fitting angle-only bound & 56.0 & 56.3 & 59.5 & 57.5 & $57.3 *$ \\
\hline Angle $=90$ & 50.6 & 51.9 & 52.5 & 54.4 & $52.4 *$ \\
\hline Best-fitting size-only bound & 93.8 & 93.0 & 92.5 & 98.8 & 94.5 \\
\hline Size $=80$ & 80.0 & 93.8 & 92.5 & 98.8 & 91.3 \\
\hline Condition VI (Integration) & Bird SI1 & Bird SI2 & Bird SI3 & Bird SI4 & Average \\
\hline$\%$ of correct responses & 73.8 & 92.5 & 93.8 & 78.8 & 84.7 \\
\hline \multicolumn{6}{|l|}{ Best-fitting bound } \\
\hline Slope & 0.20 & 0.40 & 0.40 & 0.40 & 0.35 \\
\hline Intercept & 63.75 & 45.00 & 59.25 & 45.75 & 53.44 \\
\hline \multicolumn{6}{|l|}{$\%$ of responses accounted for by } \\
\hline Best-fitting bound & 81.3 & 95.0 & 92.5 & 80.0 & 87.2 \\
\hline Best-fitting angle-only bound & 76.3 & 77.5 & 73.8 & 63.8 & $72.9 *$ \\
\hline Angle $=90$ & 70.6 & 72.5 & 70.6 & 59.4 & $68.3 *$ \\
\hline Best-fitting size-only bound & 62.5 & 71.3 & 75.0 & 70.0 & $69.7 *$ \\
\hline Size $=80$ & 59.4 & 68.1 & 69.4 & 65.6 & $65.6^{*}$ \\
\hline
\end{tabular}

Note. Bold headers indicate birds represented in Figure 4 for each condition. For ease of comparison, slope and intercept values for single-dimension categories (VA and VS) place the diagnostic dimension (angle and speed, respectively) on the Y axis. Values for the integration condition place angle on the $\mathrm{Y}$ axis. * indicates an average significantly lower than that of the average best-fitting bound for the same condition $(p<0.05)$.

\section{Decision Bound Analysis}

An estimated decision bound was computed for each individual pigeon using all categorization responses over the final 10 days of the experiment (800 trials). Those responses were compared successively to the predictions made by a series of possible linear decision bounds passing through the stimulus space. For each possible bound, the number of trials on which the bound predicted the same categorization response recorded from that pigeon was counted. The search used step sizes of 0.1 for slope and 0.25 for intercept. For each bird, the best-fitting bound was defined as that which accounted for the largest number of individual categorization responses. Tables 1 and 2 include the results of this analysis for each condition. Because step sizes were small, some searches produced more than one best fitting estimated decision bound. In those cases, the median bound is listed on the table. When there was more than one best-fitting decision bound, the accuracy of each was based on accurate categorization of the same subset of responses. 

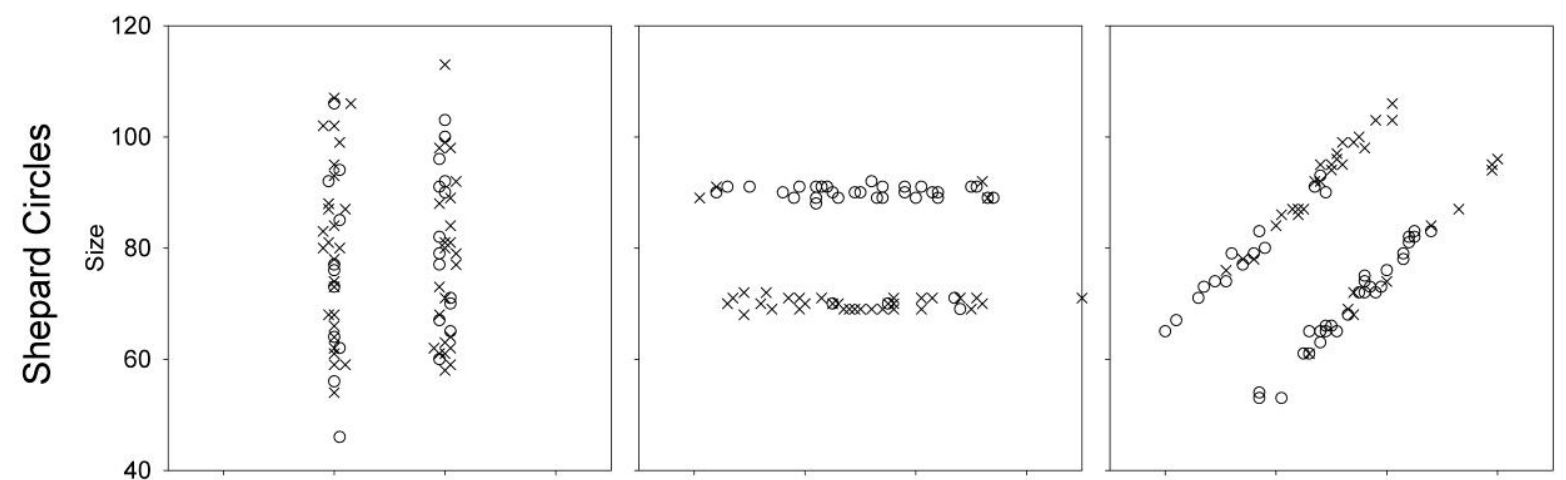

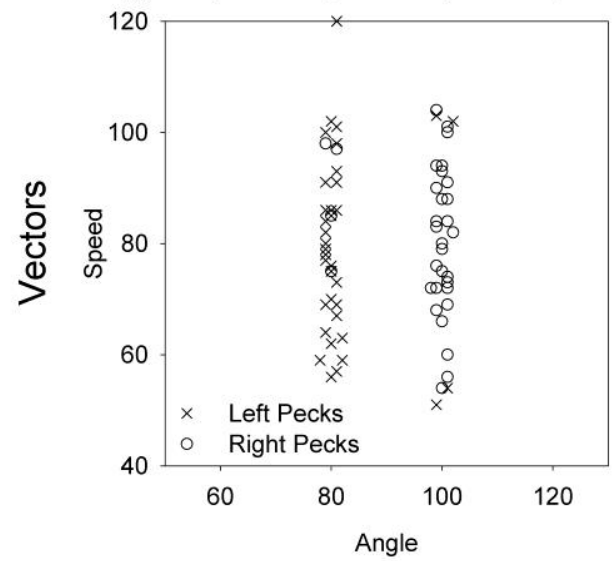

Angle Only

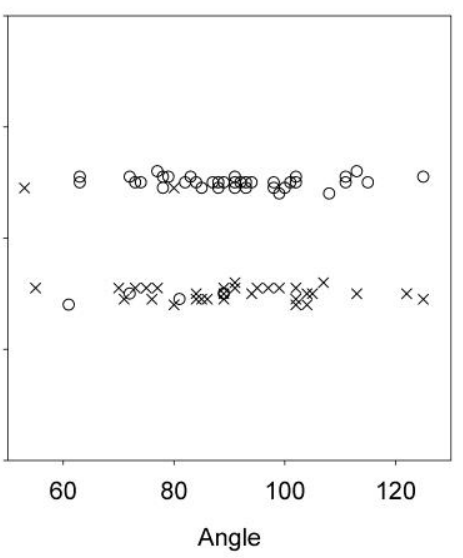

Size/Speed Only

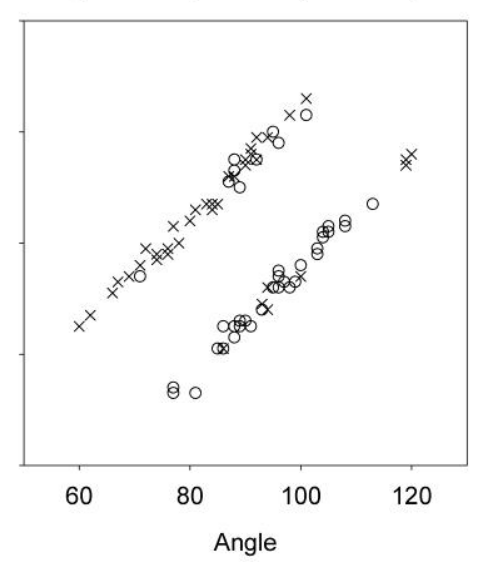

Integration

Figure 4. Final 80 categorization responses for one representative bird (see Tables 1 and 2) in each of six conditions. Circles represent right responses. Crosses represent left responses.

Individual best-fitting decision bounds for each bird and average bounds for each condition are depicted in Figure 5. The figure shows that in vector conditions, optimal decision bounds for singledimension categories were approximately perpendicular to the relevant axis, and best-fitting decision bounds for the integration condition were not perpendicular to either axis. In Shepard circle conditions, best fitting decision bounds for the size and integration conditions were both approximately perpendicular to the size axis, indicating influence from that dimension. The best-fitting decision bounds for the angleonly condition were not parallel to either axis and showed considerable variability between birds.

Tables 1 and 2 include the results of the decision-bound search. In addition to the slope, intercept, and fit of the overall best-fitting decision bounds (those depicted in Figure 5), the tables also specify the percent of responses accounted for by four additional decision bounds of interest: the optimal decision-bounds for the two single-dimension categories (angle $=90$ and size/speed $=80$ ) as well as the best-fitting angle-only and best-fitting size/speed-only decision bounds (the best-fitting bounds that were perpendicular to one axis). The information in these tables supplements overall accuracy (as depicted in Figure 3) in characterizing pigeons' categorization performance. An optimally responding bird should achieve high accuracy (percent correct), and produce a decision bound with a slope and intercept that approximates the optimal coefficients for that condition. Furthermore, the percent of correct responses should be approximately equal to the percent of responses accounted for by the best-fitting decision bound, and greater than the percent of responses accounted for by other decision bounds that do not 
reflect attention optimally allocated to the appropriate stimulus dimension or dimensions. This pattern of results reflective of optimal responding describes the performance of birds in Conditions SS, VS, VA, and VI. Note that it is also possible to produce lower accuracy while still responding consistently. For example, birds in Condition SI had relatively low percentages of correct categorizations and best-fitting decision bounds that did not match the optimal slope and intercept. Nevertheless, those best-fitting bounds accounted for a high percent of responses, greater than the percent of correct responses, indicating a consistent but inaccurate approach. Birds in Condition SA produced another, different pattern with decision bounds that did not match the optimal slope and intercept, and accounted for a low percent of responses. Note also that best-fitting decision bounds in this particular condition did not pass through the center of the stimulus space (see Figure 5), indicating that the best-fitting bounds categorized most stimuli the same way, regardless of category membership.

The pattern of responding suggested by the best-fitting decision bounds depicted in Figure 5 is further supported by analyses comparing the percent of responses accounted for by the best-fitting decision bound with the additional, single-dimension decision bounds summarized in Tables 1 and 2. In each condition, there were significant differences between the performance of the best-fitting decision bound and some of the others, all $F_{\mathrm{s}}(4,12) \geq 14.917$, all $p \mathrm{~s} \leq 0.001$. Specific differences between the best-fitting decision bound and others (all favoring the best-fitting bound) are indicated in Tables 1 and 2 with asterisks. Note that, for three of the four single-dimension conditions (SS, VS, and VA), the bestfitting decision bound is significantly better than any decision bound locked to the non-relevant dimension, and not significantly different from the optimal decision bound. Thus, birds approximated the optimal solution by selectively attending to the relevant dimension. In the fourth case of singledimension category (SA), the best-fitting decision bound did no better than either of the best-fitting single-dimension decision bounds. Thus, birds' performance was not optimal, and birds did not appear to attend to the relevant dimension any more than the irrelevant dimension. There was also an important difference between the two integration conditions. In condition VI, the best-fitting decision bound outperformed both of the best-fitting single-dimension decision-bounds. Thus, it appears that birds responded based on information from both stimulus dimensions of speed and angle. In condition SI however, the optimal decision bound was not significantly better than best-fitting size-only bound. Thus, in this case, it appears that birds were not integrating the two dimensions and were instead categorizing based exclusively on size, much like the (optimally-responding) birds in Condition SS. 

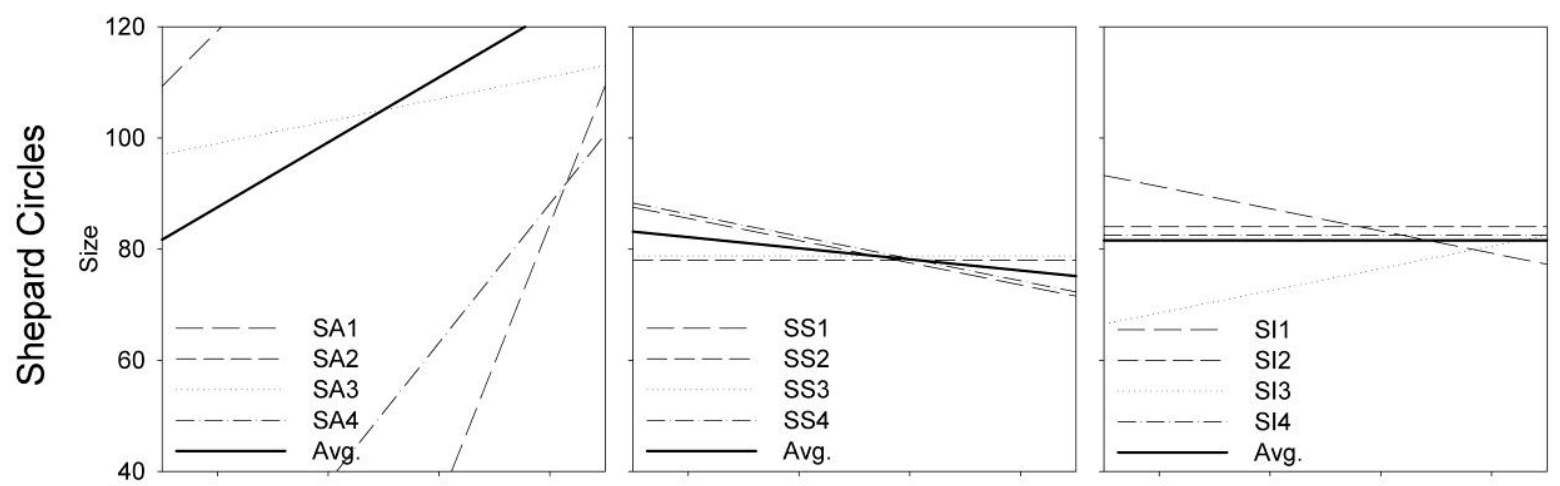

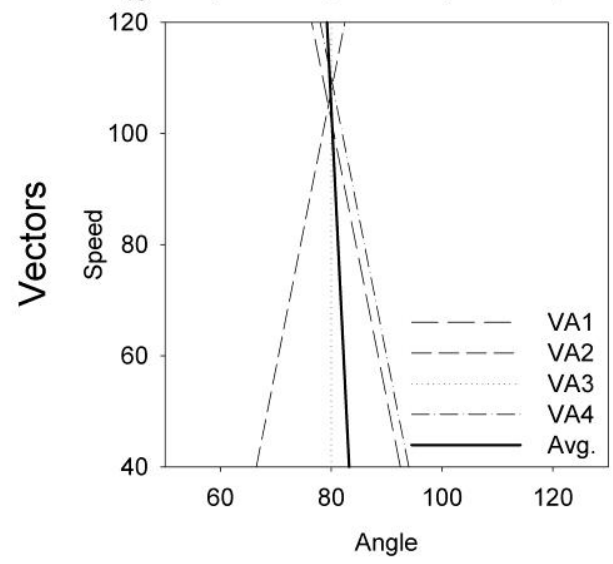

Angle Only

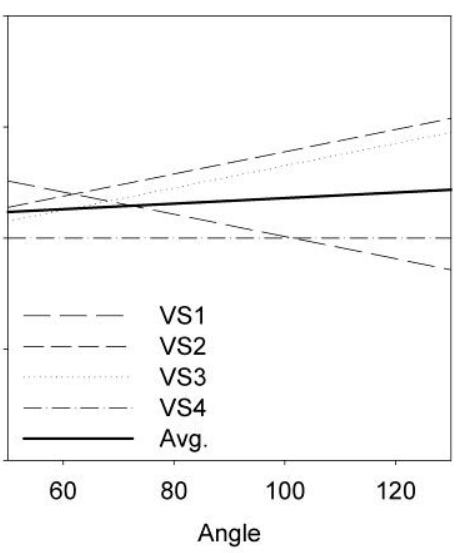

Size/Speed Only

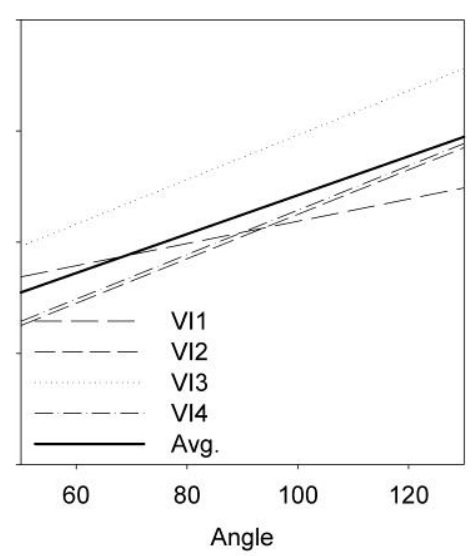

Integration

Figure 5. Best-fitting decision-bounds for each of four birds in each of six conditions (broken lines). Each solid line represents the average of all birds in a condition.

\section{Discussion}

Pigeons were better than chance at categorizing stimuli in five of six conditions, and approximated the optimal solution in four of six conditions. Specifically, they were able to categorize vectors based on angle of motion, speed, or a combination of both, and were able to accurately categorize Shepard circles based on size but not based on angle of orientation (either by itself or when combined with size). Thus, both failures to categorize optimally were due to the same factor: an inability to respond effectively based on the orientation of the radial line within Shepard circle stimuli.

Pigeons generally performed better in the vector conditions, and their success at categorizing vectors is consistent with previous research using similar stimuli (Herbranson et al., 2002). Note also that birds in the present experiment learned to accurately categorize vectors in all three conditions fairly quickly (within 60 days of training), and to similar levels of accuracy (84-91\% correct). This consistency in learning across conditions is important, because it is not always the case in categorization experiments. Ashby, Ell, and Waldron (2003), for example, found that humans learned single-dimension categories several times faster than comparable categories requiring integration of information across dimensions. This pattern is sometimes taken as evidence that humans use two cognitive systems for categorization: a faster, explicit system that is well-suited for analytic categories such as those requiring selective attention to a single dimension; and a slower, implicit system that is well-suited for non-analytic categories such as those requiring integration of information across dimensions. Smith et al. (2011), however, did not obtain the same pattern of results from pigeons. Their birds learned both kinds of categories at the same rate and 
to the same level of accuracy. Consequently, they proposed that unlike humans, pigeons learn selectiveattention and information-integration categories using a single, non-analytic categorization system. These results from vector conditions presented here are consistent with their interpretation.

At the same time, our Shepard circle conditions yielded a different pattern, with differences between conditions. Those differences are not the ones that would be predicted if pigeons used separate analytic and non-analytic categorization systems like humans. Instead, birds failed to properly learn those categories that were based entirely or in part on angle of orientation, and either performed at chance accuracy (in condition SA) or otherwise sub-optimally (in Condition SI) by responding exclusively based on another simultaneously-relevant dimension (size). This is not likely to be due to an acuity limitation, whereby the differences in angle between categories were simply too small for pigeons to discriminate, because the very same angles supported high categorization accuracy in vector conditions. If anything, one might expect that learning to discriminate angles within Shepard circles should be easier because they are constantly visible, rather than emerging over time as a stimulus moves. Furthermore, the required observation time for Shepard circles $(5 \mathrm{~s})$ was greater than the travel time of vector stimuli $(2 \mathrm{~s})$. It was also likely not due to stimulus movement drawing additional attention to vector stimuli. In some cases, stimulus movement can enhance performance on a task. Washburn (1993) for example, found that a moving sample enhanced rhesus monkeys' performance on matching to sample, presumably by drawing attention to the sample stimulus. Daniel and Katz (2016) used a similar method with pigeons and found the opposite: a negative stimulus movement effect, where movement impaired rather than enhanced performance. Furthermore, pigeons in at least one Shepard circle condition learned to categorize stationary stimuli at a level of accuracy comparable with vector conditions, indicating that under the proper circumstances pigeons can categorize stationary Shepard circles just as accurately as they can moving vectors.

Given these mixed results, we must consider why birds failed to learn some categories, when they were successful at learning other, closely-related categories. One possibility is a systematic difference in the visibility of different kinds of stimuli. That is, birds could have performed more poorly on Shepard circle conditions because something (such as motion cues or the required movement-initiating peck) drew their attention more powerfully to vector stimuli. Such an explanation is unlikely to account for these results because at least one Shepard circle condition (SS) supported accurate categorization that equaled performance on vector conditions. Furthermore, there was a required 5-second observation period for Shepard circles that was longer than the 2-second movement duration for vectors, thus providing birds with more time to process the relevant visual features. Birds generally spent the display portion of a trial pecking at the stimulus, so, on average, they would have spent more time viewing Shepard circles. Thus, the stimulus type by itself is unlikely to account for poor performance.

If not a general feature of the stimulus types, might one stimulus dimension have been more salient, drawing attention away from and/or overshadowing the other? If the radial line within a Shepard circle was less salient than the perimeter, one might expect results like those seen here. While the radial line was a different color (green versus white), the lines were comparable, with the same thickness and brightness, and the color green is well within a pigeon's visible spectrum. Thus, there is little reason to think that the visual features of radial lines made them prohibitively less salient than the perimeters that defined the size dimension. Furthermore, the radial line fell within the semicircle that defined the size dimension; consequently, it would be difficult to attend to the size of a semicircle without also viewing the portion of the display containing the radial line.

If not related to the perceptual features of the stimulus dimensions, might the failures to categorize optimally have been due to the category structures in use? The categories were intentionally designed to be easily discriminable. Although there was variability on both stimulus dimensions, that variability did not produce overlap between categories. As can be seen by the clusters of stimuli in Figure 4 (corresponding to different categories), there were no presented stimuli in the region between categories that would feature ambiguous or difficult to discriminate combinations of stimulus dimensions. This kind of structure should produce categories that are easy to discriminate, and in four of six conditions did. In the case of the remaining Conditions SA and SI, the performance-limiting angle dimension was scaled the 
same way (using precisely the same angles) as categories in the successful Conditions VA and VI. That is, the perceptual acuity required to discriminate one would necessarily be adequate for the other. If anything, one might expect vectors to be more difficult because the relevant angle is not visible at any single moment, and requires either sensory or short-term memory to reveal the diagnostic information as the stimulus moves. Finally, the differences between conditions were not a technical issue relating to the equipment or software, as human lab workers could achieve high levels of accuracy on all conditions by viewing stimuli from the pigeons' vantage point and interacting with the touch-screen manually.

Instead, we propose that failure to learn categories based on Shepard circle orientation was most likely a consequence of pigeons' adapted perceptual biases. The pigeon's aerial lifestyle may make the ability to quickly recognize objects at a variety of orientations, and from a variety of vantage points, particularly important. Hollard and Delius (1982) for example, found that pigeons did not show the standard mental rotation effect typically seen in humans. That is, they showed no decrement in response time or accuracy in a matching to sample task when comparison stimuli were presented at increasing angles of rotational disparity. If pigeons use some of the same cognitive processes in the present categorization task, it may not be surprising that they failed to appreciate different orientations between categories. Although we did not collect parallel data from human participants, humans can learn to categorize Shepard circles based on orientation, and with between-category differences even smaller than the ones used here (Ashby \& Maddox, 1990). Future research should be careful in selecting stimulus dimensions, in order to avoid categories that may be difficult or impossible for pigeons to discriminate (such as the angle-based categories of Shepard circles used here). Note though that we make no claim that pigeons are absolutely incapable of learning categories based on angle of orientation. Whereas the exact angles used were not the same, Smith et al.'s (2011) pigeons learned to categorize sine wave gratings based on angle of orientation. Thus, there may be important differences in how these two implementations of orientation (a single line pointing in various directions versus a circular, striped grating rotated into different positions) are perceived. The orientation of the long axis of an in-flight bird's body (pitch and/or yaw), for example, carries different information than the rotational position of that body (roll) relative to the ground. We cannot assume that these features, even if measured in the same way (e.g., in terms of angle), are equal in terms of category learning.

In contrast to some forms of orientation, movement may be especially important for pigeons and other animals. Ganglion cells in the pigeon retina respond selectively to specific directions of movement, indicating sensitivity to motion at one of the earliest possible points in the visual processing stream (Maturana \& Frenk, 1963). Animals with considerably simpler nervous systems similarly prioritize detection of movement. The frog's optic nerve contains "bug detectors", specifically sensitive to movement (Lettvin, Maturana, McCulloch, \& Pitts, 1968), and even flies perform impressive figureground separation based on movement (Reichardt \& Poggio, 1979). Possible reasons for the importance of motion are easy to identify, especially for pigeons. Angle of movement, for example, is vital when assessing one's own in-flight movement as well as the movement of other animals and objects. Future research might look into possible differences between categories based on the source of movement, perhaps contrasting between object-movement and self-movement. In some cases, specific kinds of movement may have particularly important implications and underlie special kinds of categories (such as in the case of biological movement; Dittrich, Lea, Barrett, \& Gurr, 1998).

In conclusion, categorization is not only a fundamental cognitive process in and of itself, but also one that relies on a variety of other contributing abilities, including perception and attention. The present research indicates one way that these contributing processes may affect how pigeons categorize complex stimuli, resulting in categorization performance that is best suited to their lifestyle and ecological niche, and meaningfully different from the categorization performance of humans. These findings also emphasize the importance of using divergent methods, stimulus types, and species in research on categorization, as well as the broad relevance of categorization to a host of cognitive processes, and the mechanisms behind them. 


\section{References}

Ashby, F. G., Ell, S. W., \& Waldron, E. M. (2003). Procedural learning in perceptual categorization. Memory \& Cognition, 31, 1114-1125.

Ashby, F. G., \& Gott, R. (1988). Decision rules in the perception and categorization of multidimensional stimuli. Journal of Experimental Psychology: Learning, Memory and Cognition, 14, 33-53.

Ashby, F. G., \& Maddox, W. T. (1990). Integrating information from separable psychological dimensions. Journal of Experimental Psychology: Human Perception \& Performance, 16, 598-612.

Ashby, F. G., \& Maddox, W. T. (1992). Complex decision rules in categorization: Contrasting novice and experienced performance. Journal of Experimental Psychology: Human Perception and Performance, 18, 50-71.

Brown, P. L., \& Jenkins, H. J. (1968). Autoshaping of the pigeon's keypeck. Journal of the Experimental Analysis of Behavior, 11, 1-8.

Chase, S., \& Heinemann, E. G. (1972). Choices based on redundant information: An analysis of two-dimensional stimulus control. Journal of Experimental Psychology, 92, 161-175.

Daniel, T. A., \& Katz, J. S. (2016). A negative stimulus movement effect in pigeons. Behavioural Processes, 130, 11-18.

Dittrich, W. H., Lea, S. E. G., Barrett, J., \& Gurr, P. R. (1998). Categorization of natural movements by pigeons: Visual concept discrimination and biological motion. Journal of the Experimental Analysis of Behavior, 70, 281-299.

Herbranson, W. T., Fremouw, T., \& Shimp, C. P. (1999). The randomization procedure in the study of categorization of multidimensional stimuli by pigeons. Journal of Experimental Psychology: Animal Behavior Processes, 25, 113-135.

Herbranson, W. T., Fremouw, T., \& Shimp, C. P. (2002). Categorizing a moving target in terms of its speed, direction, or both. Journal of the Experimental Analysis of Behavior, 78, 249-270.

Herrnstein, R. J., \& Loveland, D. H. (1964). Complex visual concepts in the pigeon. Science, 146, 549-551.

Hollard, V. D., \& Delius, J. D. (1982). Rotational invariance in visual pattern recognition by pigeons and humans. Science, 218, 804-806.

Jitsumori, M., \& Delius, J.D. (2001). Object recognition and object categorization in animals. In T. Matsuzawa (Ed.), Primate origins of human cognition and behavior (pp. 269-293). Tokyo: Springer-Verlag.

Lea, S. E. G., Wills, A. J., Leaver, L. A., Ryan, C. M. E., Bryant, C. M. L., \& Millar, L. (2009). A comparative analysis of categorization of multidimensional stimuli: II. Strategic information search in humans (Homo sapiens) but not pigeons (Columba livia). Journal of Comparative Psychology, 123, 406-420.

Lettvin, J. Y., Maturana, H. R., McCulloch, W. S., \& Pitts, W. H. (1959). What the frog's eye tells the frog's brain. Proceedings of the IRE, 47, 1940-1951.

Lockhead, G. R. (1966). Effects of dimensional redundancy on visual discrimination. Journal of Experimental Psychology, 72, 95-104.

Maddox, W. T., Ashby, F. G., \& Bohil, C. J. (2003). Delayed feedback effects on rule-based and informationintegration category learning. Journal of Experimental Psychology: Learning, Memory, and Cognition, 29, 650-662.

Maddox, W. T., \& Dodd, J. L. (2003). Separating perceptual and decisional attention processes in the identification and categorization of integral-dimension stimuli. Journal of Experimental Psychology: Learning, Memory, and Cognition, 29, 467-480.

Maturana, H. R., \& Frenk, S. (1963). Directional movement and horizontal edge detectors in the pigeon retina. Science, 142, 977-979.

Poling, A., Nickel, M., \& Alling, K. (1990). Free birds aren't fat: Weight gain in captured wild pigeons maintained under laboratory conditions. Journal of the Experimental Analysis of Behavior, 53, 423-424.

Reichardt, W., \& Poggio, T. (1979). Figure-ground discrimination by relative movement in the visual system of the fly. Biological Cybernetics, 35, 81-100.

Shepard, R. N. (1964). Attention and the metric structure of the stimulus space. Journal of Mathematical Psychology, 1, 54-87.

Smith, J. D., Ashby, F. G., Berg, M. E., Murphy, M. S., Spiering, B., Cook, R. G., \& Grace, R. C. (2011). Pigeons' categorization may be exclusively nonanalytic. Psychonomic Bulletin \& Review, 18, 414-421.

Smith, J. D., Berg, M. E., Cook, R. G., Murphy, M. S., Crossley, M. J., Boomer, J., ...Grace, R. C. (2012). Implicit and explicit categorization: A tale of four species. Neuroscience \& Biobehavioral Reviews, 36, 2355-2369. 
Washburn, D. A. (1993). The stimulus movement effect: Allocation of attention or artifact? Journal of Experimental Psychology: Animal Behavior Processes, 19, 380-390.

Wills, S. E., Lea, S. E. G., Leaver, L. A., Osthaus, B., Ryan, C. M. E., Suret, M. B., ...Millar, L. (2009). A comparative analysis of the categorization of multidimensional stimuli: I. Unidimensional classification does not necessarily imply analytic processing; Evidence from pigeons (Columba livia), squirrels (Sciurus carolinensis), and humans (Homo sapiens). Journal of Comparative Psychology, 123, 391-405. 